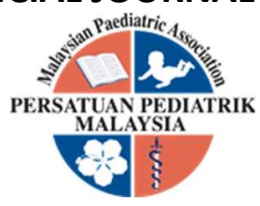

\title{
A RARE PRESENTATION OF CONGENITAL ARYEPIGLOTTIC CYST WITH CONCURRENT LARYNGOMALACIA
}

\author{
Yi Wee Lim, Hazama Mohamad, Suzina Sheikh Ab Hamid, Azliana Aziz
}

\begin{abstract}
Congenital laryngeal cyst remains a rare but possible cause of airway obstruction that requires urgent referral to an otorhinolaryngology centre. Vallecular cyst commonly present immediately after birth with variable degree of airway obstruction symptoms depending on its size, but presentation of aryepiglottic cyst with concurrent laryngomalacia remains a conundrum as there are very few cases reported. Unlike the more obvious manifestation of stridor or increasing respiratory effort in most supraglottic cyst or laryngomalacia, the neonate presented only with weak cry which masqueraded early suspicion and referral of an airway pathology. We provide discussion on the rare clinical presentation aryepiglottic cyst with synchronous laryngomalacia and its management.
\end{abstract}

Keywords: Laryngeal Cyst, Aryepiglottic Cyst, Laryngomalacia, Deroofing, Marsupialization DOI: $10.51407 /$ mjpch.v28i1.147

\section{Introduction}

Laryngeal cyst is a congenital cause of stridor in the neonate and can often be misdiagnosed as laryngomalacia. Most common laryngeal cyst reported are vallecular cyst. The manifestation of a supraglottic cyst at the aryepiglottic fold with synchronous manifestation of laryngomalacia is extremely rare. We report a case of a term neonate who otherwise just presented with symptoms of weak cry since birth with soft stridor only during feeding without apparent respiratory distress. On examination, the child was comfortable at rest. She had a strenuous and weak cry with intermittent soft inspiratory stridor while crying without apparent hoarseness. A bedside flexible laryngoscopy was performed initially reviewing the presence of a supraglottic cyst with significant obstruction to the laryngeal inlet. A direct laryngoscopy, was performed under general anesthesia confirming the location of the cyst at the right aryepiglottic fold and presence of concomitant bilateral short aryepiglottic fold. Endoscopic deroofing of the cyst and release of the contralateral aryepiglottic fold was performed. The patient developed recurrent poor cry again 2
Received: 20 February 2021; Accepted revised manuscript: 12 January 2022; Published online: 26 January 2022

months after the initial surgery. A repeated bedside flexible laryngoscopy showed recurrent swelling at the previous aryepiglottic cyst site. The patient subsequently underwent an endoscopic marsupialization of the cyst and remain symptomfree after 11 months after surgery.

\section{Case Report}

A 10-day-old neonate was referred to Otorhinolaryngology Clinic from the Emergency Department with the preliminary diagnosis of laryngomalacia. The child was born full-term with a birth weight of $2.9 \mathrm{~kg}$ and delivered via vaginal delivery uneventfully. There were no post delivery complication and child was discharged home well. Mother noted child had very weak cry since birth

Department of Otorhinolaryngology-Head \& Neck Surgery, School of Medical Sciences, Universiti Sains Malaysia Health Campus, 16150 Kubang Kerian, Kelantan, Malaysia

\section{Corresponding Author:}

Azliana Aziz, Department of Otorhinolaryngology-Head \& Neck Surgery, School of Medical Sciences, Universiti Sains Malaysia Health Campus, 16150 Kubang Kerian, Kelantan, Malaysia

Tel: +609-7676420 Email: az_aziz@usm.my 
and this became more apparent after about a week at home. Occasionally, the mother noted child developed interrupted feeding and child were not able to tolerate prolonged breast feeding.

On examination, she appeared comfortable at rest and was able to maintain an oxygen saturation of 9699\% under room air. There was no gross craniofacial abnormalities, her nasal patency was clear. Her cry was strenuous and weak without hoarseness. Soft inspiratory stridor with moderate subcostal and substernal recession was noticeable only during effortful cry. A flexible laryngoscopy at bedside showed there was a large swelling at the left supraglottis causing partial narrowing of the airway. The complete visualisation of the laryngeal inlet was made difficult by the posteriomedial deflection of epiglottis and the vocal cords mobility were normal albeit unable to fully visualised the anterior commisure. The child underwent direct laryngoscopy and bronchoscopy on day 13 of life under general anaesthesia. Intubation was assisted with the guide of pediatric C-Mac laryngoscope and there was no difficulty in intubating the child. Under suspension laryngoscopy with a Parsons laryngoscope size 4 and a Hopkins straight rigid telescope, a cystic mass was seen at the left aryepiglottic fold partially obscuring the laryngeal inlet. The cyst was obscuring the view of both vocal folds (Figure 1A). The cyst was deroofed on the medial surface using a $2 \mathrm{~mm}$ laryngeal micro scissor in which clear fluid content was suctioned out cautiously. After clearing the content of cyst, we found that the right aryepiglottic fold was short, there was still limited view of bilateral anterior commisure, and the short aryepiglottic fold was still pulling the epiglottis posteromedially. We proceeded with bilateral aryepiglottic fold release using both a right and left turned micro scissors. Post excision, there was better visualization of the glottic inlet and both vocal cords (Figure IB). The child was kept intubated for 48 hours and extubated without any respiratory symptoms.

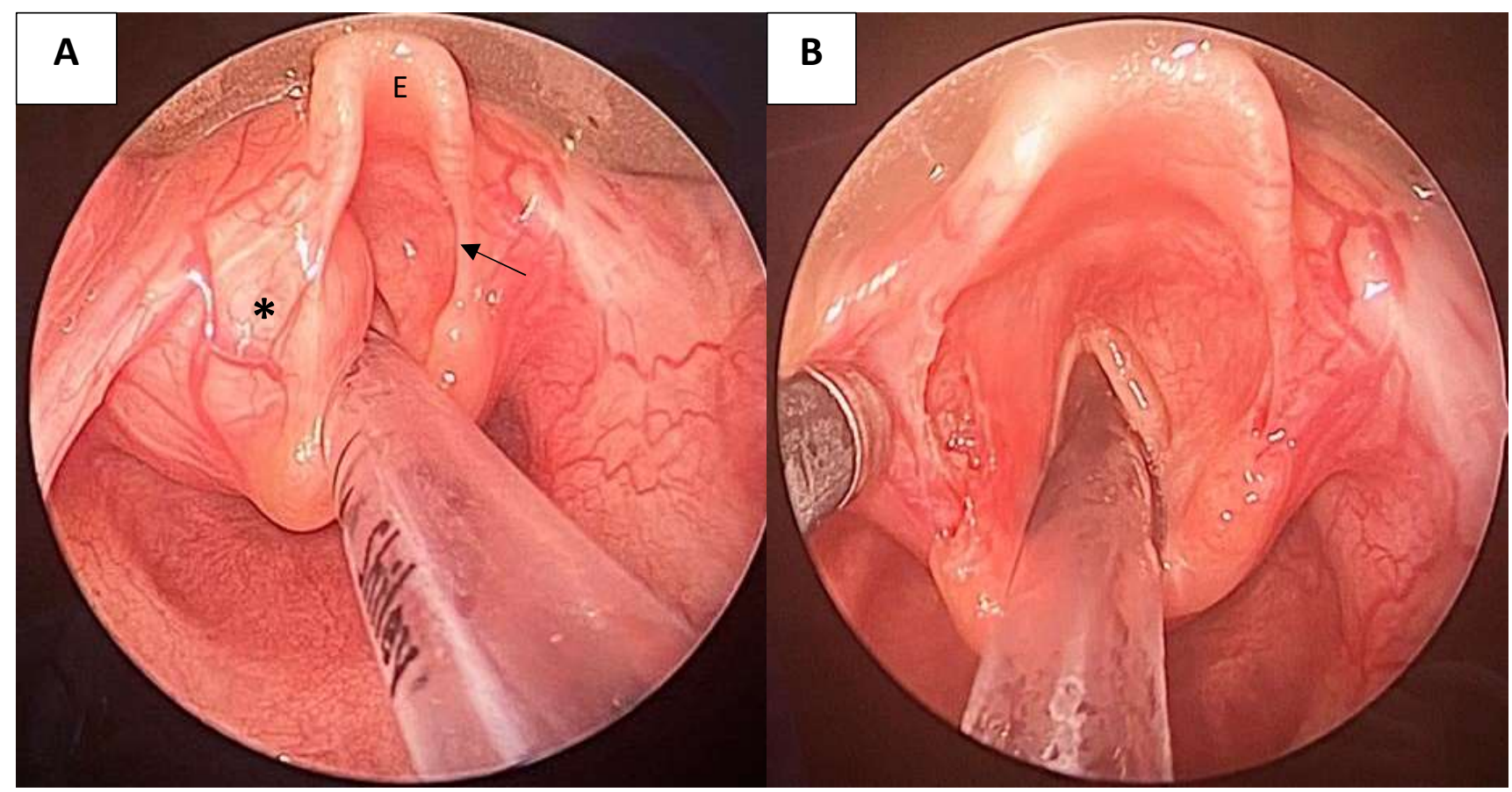

Figure 1. Direct laryngoscopy view of laryngeal cyst

(A) Endoscopic findings prior to marsupialization of cyst. Large left AE fold cyst depicted as *, E - epiglottis and arrow showing a short right AE fold. (B) Post endoscopic deroofing. 
Postoperatively, the child initially regains a stronger cry. However, 2 months after surgery, the child developed recurrent symptoms of weak cry. This time, there was no noisy breathing and no interrupted feeding. A repeated bedside flexible laryngoscopy revealed a recurrent swelling at the same site of the previous aryepiglottic cyst. The child underwent a transoral endoscopic marsupialization of the aryepiglottic cyst. Intraoperatively, the cyst was attached in a broad-based fashion to the lateral aspect of the epiglottis, into the vallecula, along the free margin and lateral surface of the aryepiglottic. Therefore, the cyst was decompressed by incising the upper surface of the cyst with straight laryngeal microscissor to reveal viscous clear fluid content, and this facilitated the removal of the remaining cyst wall lining (Figure 2) using a laryngeal microdebrider. Postoperatively, the child was adequately covered with broad-spectrum antibiotic and intravenous dexamethasone. The child was extubated after 48 hours of observation. There were no signs of upper airway obstruction and the child was discharged home 3 days after surgery.

Currently, the child is 11 months post-surgery and remains well with a good cry and no more noisy breathing. Histological examination showed soft tissue lined by squamous epithelium, consistent with a cyst.

\section{Discussion}

A congenital laryngeal cyst is a rare cause of stridor in neonates. The annual incidence of congenital laryngeal cyst is estimated to be only about 1.82 per 100000 live birth [1]. The location of laryngeal cyst presenting at the aryepiglottic fold in our case is rare since majority of laryngeal cyst arises at the vallecula and the saccule of ventricle while only $2.2 \%$ are found at the aryepiglottic fold [2]. Additionally, synchronous presentation of aryepiglottic cyst with laryngomalacia is extremely rare with only few cases ever reported [3,4] and this coexistence causes increasing upper airway obstruction which severity was masqueraded by a soft and weak cry.

Histologically, this congenital laryngeal cyst can be further divided into either ductal or saccular cyst based on its location and surface mucosa [2]. Ductal cyst being the more common type arises from fluid accumulation in the setting of the obstructed submucous gland. Saccular cysts develop from embryological malformation or atresia of the laryngeal ventricle which is most likely in the case of congenital laryngeal cyst.

The aryepiglottic cyst usually presents with loud stridor due to a "ball-valve" effect during inspiration as with any other supraglottic masses [5]. Depending on the size, the large cyst can cause severe respiratory distress and complicating intubation.

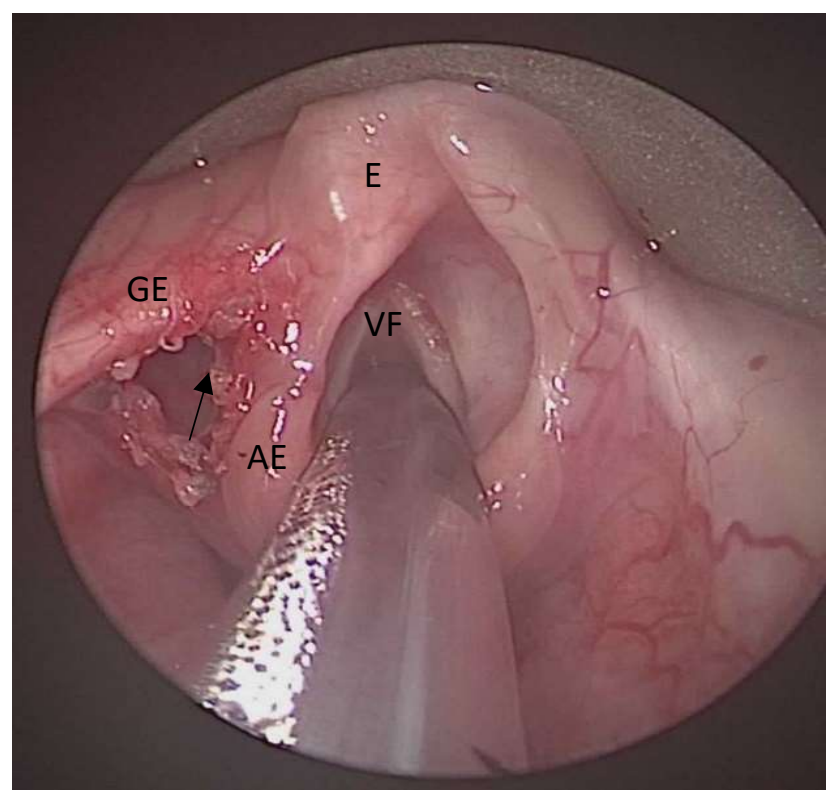

Figure 2. Post revision surgery with arrow showing marsupialization of the entire superior cyst wall GE - left lateral glossoepiglottic fold, E - epiglottis, AE - left aryepiglottic fold, VF- vocal fold 
In our case, the presence of a large cyst at the aryepiglottic fold did not result in obvious respiratory distress but manifested mildly as a weak cry with intermittent soft stridor during feeding. This has avoided the need for tracheostomy in our child. Such subtle symptoms could also be present in congenital vocal cord palsy and therefore emphasizing the need to perform a bedside laryngoscopy to confirm the diagnosis.

Direct laryngoscopy remains the gold standard for diagnoses of the laryngeal cyst as it allows for a clear demonstration of origin, extension and palpation of the mass [6]. In our opinion, direct laryngoscopy is adequate to establish a diagnosis but other imaging modalities may be helpful especially in centres without otorhinolaryngology expertise. Computed Tomography (CT) or Magnetic Resonance Imaging (MRI) are preferred as they can depict the location and the extension of the cyst. Ultrasound has also been reported to be useful to diagnose congenital laryngeal cyst during antenatal screening [7]. Cervical ultrasonography in laryngeal cyst can demonstrate sonographic evidence of its cystic nature with plain doppler and most importantly differentiate a vascularized mass such as a hemangioma using flow void Doppler [8].

There is no consensus for the optimal surgical method owing to this rare disease. Lifesaving tracheostomy may be performed first while awaiting definitive surgery. The coexistence of laryngomalacia with aryepiglottic cyst in our case warrants for urgent surgical intervention. Definitive surgery involves either endoscopic or external approach. The commonly employed technique is the endoscopic marsupialization of laryngeal cyst is favoured due to its low recurrence rate [9]. Additionally, carbon dioxide (CO2) laser has been used with endoscopic marsupialization to vaporize the cyst lining [10]. Needle aspiration may be used for decompression of the cyst to alleviate airway obstruction and to achieve better visualization of the surgical field but is not recommended as the sole surgical procedure as this method does not address the blocked submucous gland which will continue to secrete mucus leading to recurrence. The external approach can be reserved for a larger cyst or those which recur following endoscopic removal.

We prefer endoscopic marsupialization using cold instrument over complete excision of the cyst wall with $\mathrm{CO} 2$ laser as this method has the advantage of avoiding thermal injury to the surrounding mucosa. The use of coblator may also help to tackle intraoperative bleeding. In our case, we learned that endoscopic deroofing of laryngeal cyst is inadequate as this procedure does not address the underlying pathophysiology of blockage of the underlying submucous gland and would only provide temporal relief of the cystic compression at the supraglottis.

Supraglottoplasty was required in our case due to the severity of the laryngomalacia and the location of the cyst. There are case reports $[3,4]$ which demonstrated improvement of symptoms by excision of the cyst alone without supraglottoplasty but those are mainly reported in vallecular cyst. To our knowledge, there has not been any cases reported of concomitant aryepiglottic cyst with laryngomalacia let alone discussion on options of conservative or surgery for the coexisting laryngomalacia. We decided to proceed with aryepiglottoplasty since the right aryepiglottic fold were in part of the surgical site of the cyst. Further a left aryepiglottoplasty was performed to ensure a more symmetrical position of the epiglottis.

\section{Conclusion}

We would like to highlight a few important lessons learned from our case. First, not all congenital upper airway obstruction presents with stridor. A weak cry should also arouse the suspicion of the clinician for any possible cause of upper airway obstruction. Second, congenital laryngeal cyst and laryngomalacia can coexist in the same patient. Addressing the short aryepiglottic cyst in our case had improved symptoms of interrupted feeding and stridor even after developing recurrent cyst. Lastly, when meeting with a congenital laryngeal cyst, endoscopic deroofing may be attempted as the initial measure to decompress thereby establishing the airway and avoiding tracheotomy but a proper marsupialization or excision of the cyst should be followed shortly to prevent recurrence of the cyst. 


\section{References}

1. Pak MW, Sang Woo JK, van Hasselt CA. Congenital laryngeal cysts: current approach to management. The Journal of Laryngology \& Otology. 1996;110:8546.

2. Arens $\mathrm{C}$, Glanz H, Kleinsasser O. Clinical and morphological aspects of laryngeal cysts.

European Archives of Oto-Rhino-Laryngology. 1997;254:430.

3. Gandhi S, Raza S A, Thekedar P, Mishra P. Congenital vallecular cyst with laryngomalacia: $A$ report of two cases. J Laryngol Voice 2011;1:27-9

4. Ku AS. Vallecular cyst: report of four cases-one with co-existing laryngomalacia. J Laryngol Otol. 2000 Mar;114(3):224-6.

5. Vaibhav A K, Richard B L, Jeffrey B G. Unanticipated Difficult Intubation as a Result of an Asymptomatic Vallecular Cyst. Anesthesiology 1999; $\quad 91: 872$

DOI: 10.1097/00000542-199909000-00043

6. Abd Aziz A, Abdullah AF, Ahmad RAR. Two cases of congenital vallecular cyst: a reminder of the potentially fatal cause of upper airway obstruction in infants. Malays J Med Sci. 2010;17:68-73.

7. Cuillier F, Samperiz S, Testud R, Fossati P. Antenatal diagnosis and management of a vallecular cyst. Ultrasound in obstetrics \& gynecology : the official journal of the International Society of Ultrasound in Obstetrics and Gynecology 2002; 20: 623-626. 2002/12/21. DOI: 10.1046/j.1469-0705. 2002.00860.x

8. Shita L, Rypens F, Hassid S, Vermeylen D, Struyven J. Sonographic demonstration of a congenital laryngeal cyst. Journal of ultrasound in medicine: official journal of the American Institute of Ultrasound in Medicine 1999; 18: 665-667. DOI: 10.7863/jum.1999.18.9.665.

9. Suzuki J, Hashimoto S, Watanabe K, Takahashi K. Congenital vallecular cyst in an infant: case report and review of 52 recent cases. The Journal of Laryngology \& Otology 2011; 125: 1199-1203. DOI: $10.1017 /$ S0022215111001368.

10. Massoth LJ and Digoy GP. Flexible carbon dioxide laser-assisted endoscopic marsupialization and ablation of a laryngeal saccular cyst in a neonate. The Annals of Otology, Rhinology, and Laryngology. 2014;123:541-4. 